\title{
Historique de la colonisation du milieu de la pres- qu'île d'Ampasindava : transformations du paysage et système de conservation
}

\author{
Jacquis Andonahary Tahinarivony', Niry Solofonianja \\ Rasoanaivo', Patrick Ranirison!, Nathalie Rasolofo", \\ Laurent Gautier"
}

\author{
Correspondence: \\ Jacquis Andonahary Tahinarivony \\ Département de Biologie et Ecologie Végétales, Université \\ d'Antananarivo, Madagascar \\ E-mail: andonahary@yahoo.fr
}

\begin{abstract}
In the northwest of Madagascar, the Ampasindava peninsula is home to the essential part of what remains of the forests of the Sambirano phytogeographic domain. The area has great importance in terms of biogeography, ecology, and socio-cultural aspects. The first documented human presence dates back to the tenth century, during the development of the maritime trade in Madagascar, before becoming the first headquarters of the Sakalava kingdom in the northwest of Madagascar. Several facts contributed to the degradation of the landscape, like the practice of the tavy and the war between Bemihisatra and Merina. In the peninsula, the western part was the most affected, on contrary to the eastern part where are located the forest massifs which served as refuges during the tribal wars. After the annexion by France, of an important territory including the peninsula (August 6th, 1896), the Merina left the region, and these forest massifs acquired a sacred status and as such were protected against destruction, preserving the characteristics of the primary forests of Sambirano. Elsewhere, a process of natural reconstruction of the vegetation started. These historic facts lead us to conclude that the forests of Dypsis spp. (Arecaceae) and Sarcolaenaceae are not primary resulting from a difference in substrate or climate, but are to be interpreted as old secondary forests, deriving from the long-term process of reconstruction of the vegetation. For several years, the analysis of satellite imagery demonstrates that the rhythm of the tavy has dramatically increased in the region. This increase stems not only from a population growth, but also from a growing pressure for land to generate income for the purchase of manufactured products. The practice of the tavy represents a major threat to forests and different types of residual natural habitats. Secondary forests are the most sensitive and most suitable for rice production. Primary sacred forests have remained so far untouched. Their traditional protection is however recently shaken by the influx of immigrants, who show little respect to traditional ban.
\end{abstract}

\section{RÉSUMÉ}

Dans le Nord-ouest de Madagascar, la presqu'île d'Ampasindava abrite une partie essentielle des restes des forêts du domaine du Sambirano. La région possède une grande importance tant biogéographique et écologique, que socio-culturelle. Elle a connu au X siècle sa première implantation humaine et il est vraisemblable que l'Homme l'ait parcourue et utilisée régulièrement à l'époque du développement du réseau maritime à Madagascar, avant que le Sambirano ne devienne le premier siège du royaume Sakalava dans le Nord-ouest de l'île. Plusieurs faits ont contribué à la déstructuration du paysage écologique initial, comme la pratique de l'abattis sur brûlis ou tavy et les guerres entre les Bemihisatra de la région et l'armée du royaume Merina. Si la zone Ouest de la région a été la plus touchée, celle de l'Est où se trouvent les grands massifs forestiers a servi de refuges durant ces guerres. Après I'annexion par la France, le 6 août 1896, d'un important territoire comprenant la presqu'île, les Merina ont quitté la région, et ces massifs forestiers acquirent un caractère sacré et furent ainsi protégés contre la destruction et le défrichement en conservant les caractéristiques des forêts climaciques intactes du Sambirano. Ailleurs, un processus de reconstitution naturelle de la végétation se mit en route. Ces divers aspects historiques permettent d'avancer que les forêts à Sarcolaenaceae et à Dypsis spp (Arecaceae), avec leurs caractéristiques physionomiques, structurales et floristiques, ne découlent pas tant de caractéristiques du substrat ou du climat, mais seraient plutôt des forêts secondaires âgées, issues du long processus d'évolution progressive du dynamisme de la succession végétale. Depuis quelques années, I'analyse des images satellite montre que le rythme auquel le tavy est pratiqué s'intensifie dans la région. Cet accroissement découle non seulement de la croissance démographique, mais aussi d'un besoin accru en terres pour générer les revenus nécessaires à l'achat de produits manufacturés. La pratique du tavy représente une des principales menaces pour les forêts et les différents types d'habitats naturels résiduels. Les forêts secondaires âgées qui sont si- 


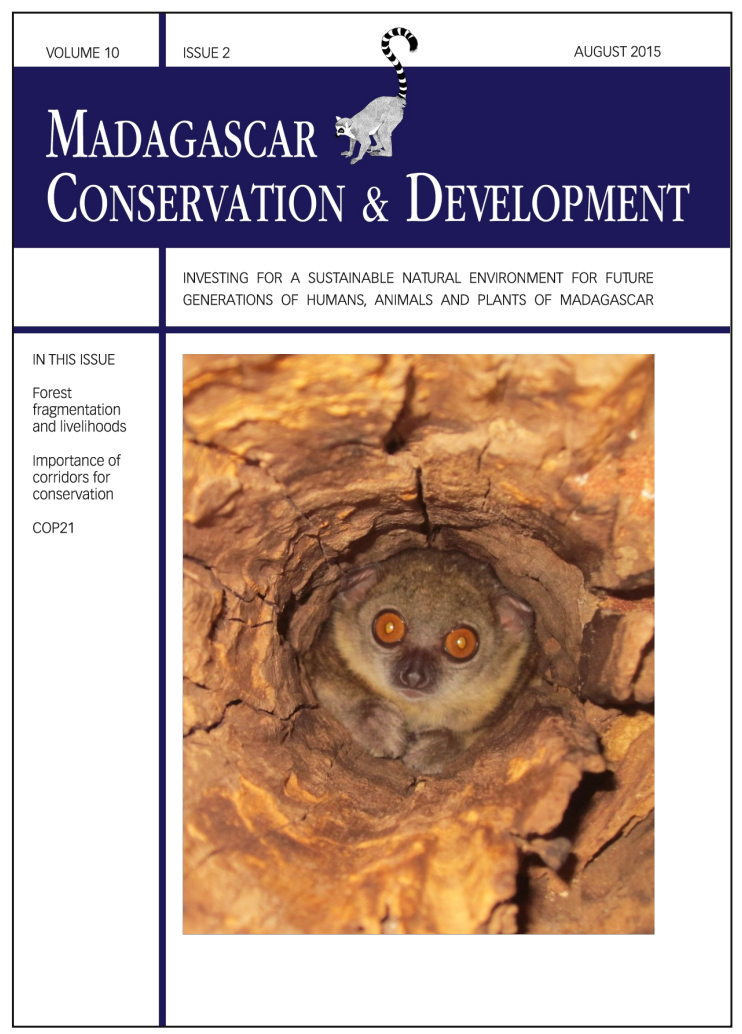

Madagascar Conservation \& Development is the journal of Indian Ocean e-Ink. It is produced under the responsibility of this institution. The views expressed in contributions to MCD are solely those of the authors and not those of the journal editors or the publisher.

All the Issues and articles are freely available at http://www.journalmcd.com

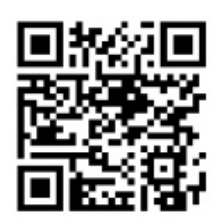

Contact Journal MCD

info@journalmcd.net for general inquiries regarding MCD funding@journalmcd.net to support the journal

Madagascar Conservation \& Development Institute and Museum of Anthropology

University of Zurich

Winterthurerstrasse 190

$\mathrm{CH}-8057$ Zurich

Switzerland

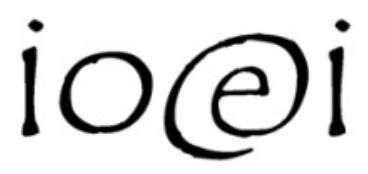

Indian Ocean e-Ink

Promoting African Publishing and Education

www.ioeink.com

Missouri Botanical Garden (MBG)

Madagascar Research and Conservation Program BP 3391

Antananarivo, 101, Madagascar 
tuées hors des zones traditionnellement protégées sont les plus sensibles car elles sont les plus propices à la production de riz. Les forêts des massifs sacrés, en revanche, ne semblent pas encore réellement menacées mais leur protection traditionnelle est quelque peu ébranlée depuis par l'arrivée récente et massive d'immigrants qui respectent peu les interdits traditionnels.

\section{INTRODUCTION}

Pour sa méga-diversité inégalable, Madagascar est considéré comme un 'joyau écologique' ou un 'sanctuaire de la nature' (Joseph-Philibert Commerson In Oberlé 1981 : i). Malheureusement, on observe une dégradation progressive de ses écosystèmes depuis l'introduction du riz et de son système de production, l'agriculture itinérante sur brûlis ou tavy, par les proto-Indonésiens. Cette pratique a fait disparaître une grande partie de la forêt, différents types d'habitats et plusieurs espèces animales et végétales majoritairement endémiques de l'île (Goodman et Benstead 2005). Elle est un problème permanent pour le pays, tout comme la pauvreté. Mais c'est justement cette pauvreté qui explique en partie le phénomène de la destruction de la nature, et notamment la déforestation. La plupart des activités de la population se pratique encore sur un mode traditionnel, souvent destructif et très peu rentable. La partie orientale malgache est d'ailleurs connue pour être depuis de longues années un terrain de recherches et d'expérimentations pour trouver et appliquer des alternatives proposées par différentes entités afin de remédier à ce problème.

Le tavy est une des causes principales de la déforestation à Madagascar (Scales 2014). Flacourt (1661: 23) en donne une des premières descriptions au fil de ses observations dans la partie orientale de Madagascar : " Ils plantent leurs riz dans les montagnes et les vallées, après avoir coupé les bois. Lorsque ces bois sont brûlés, toute la terre est couverte de cendres lesquelles se détrempent par la pluie, et au bout de quelque temps ils sèment le riz ». En 1947, Pierre Gourou a alors défini l'agriculture itinérante sur brûlis comme une technique commune aux régions chaudes humides et une solution trouvée par les agriculteurs aux problèmes que leur posaient le sol et le relief (Veyret 1948). Confrontés aux conséquences du tavy sur la déforestation, les paysans se justifient par l'idée qu'en l'absence de plaines cultivables, c'est la seule technique adaptée pour la culture (Vicariot 1970). Dans le cadre de ce travail, la définition du tavy se rapproche de I'utilisation locale du terme, qui désigne à la fois le système cultural par abattis-brûlis, mais aussi la parcelle de culture. La région Nord-ouest de Madagascar, notamment le domaine du Sambirano, n'échappe pas à la problématique du tavy car il est central dans l'économie des paysans, utilisé non seulement pour la production du riz mais aussi comme un moyen d'appropriation des terrains (Aubert et al. 2003, Razafy Fara 2004).

Humbert (1955) a rattaché la forêt climacique de la presqu'île d'Ampasindava au domaine phytogéographique du Sambirano, en la définissant comme un type de forêt dense humide sempervirente de basse altitude. Dans sa cartographie de la végétation de Madagascar basée sur les missions photographiques des années 1950 (Humbert et Cours-Darne 1965), il constate une déforestation ancienne de la majorité de la presqu'île à l'exception de quelques massifs centrés sur des montagnes. Dans une cartographie plus récente également menée au niveau de l'île dans son ensemble sur la base de l'imagerie satellitaire (Moat et Smith 2007), les forêts sempervirentes ont nettement régressé. Ce qui est paradoxal, c'est que d'importantes surfaces de zones carto- graphiées comme des forêts humides secondaires par Humbert et Cours Darne (1965) correspondent à des zones classées comme des forêts sèches occidentales. II est important de rappeler que le signal visible des forêts sèches peut parfois être confondu avec celui des faciès de dégradation des forêts humides, particulièrement à l'échelle considérée et si on n'a pas la possibilité de comparer des images de saison sèche à celles de saison humide. Tout en soulignant le remarquable effort, fruit de travaux antérieurs pionniers (Du Puy et Moat 1998), il est par ailleurs inévitable qu'un travail de cette ampleur se soit heurté à une pénurie de données au sol. A notre connaissance, aucune vérification de terrain n'a été menée dans la presqu'île et nous avons tout lieu de penser que la cartographie climatique de cornet (1974) a joué un grand rôle dans cette interprétation. Malheureusement, cette cartographie se base sur un réseau très lâche de stations climatiques.

Lors de deux campagnes de quatre mois chacune menées entre 2008 et 2010 dans la presqu'île pour en décrire la flore et la végétation, ainsi que lors de missions plus courtes par la suite, nous avons pu faire les constatations suivantes: (i) les forêts denses humides sempervirentes climaciques de superficie importante ne sont effectivement plus rencontrées qu'au niveau des massifs sacrés. Ceux-ci sont entourés par divers types de formations secondaires, de jachères et de friches (Tahinarivony 2014) ; (ii) la région ouest est occupée par des forêts caractérisées par une composition floristique différente, à Sarcolaenaceae et Dypsis spp. (Arecaceae) (Tahinarivony 2014) ; (iii) aucune espèce caractéristique des forêts sèches occidentales n'a été trouvée dans cette zone (Ammann 2011) ; (iv) on constate toujours dans ces forêts une proportion de ligneux issus de rejets de souche anormale pour une végétation climacique, ainsi que la présence de nombreux taxons de la reconstitution des forêts secondaires ; et (V) on y trouve régulièrement des lambeaux de forêts denses humides, aux endroits les moins propices à la culture (ravins encaissés ou dominance d'affleurements rocheux) et témoignent vraisemblablement de leur étendue généralisée par le passé.

Nous mettons ainsi en doute l'interprétation de Moat et Smith (2007). Toutes nos observations concourent à considérer que l'interprétation de Humbert et Cours Darne (1965) était la bonne. Dans cette contribution, nous nous proposons d'analyser I'histoire de la presqu'île pour voir dans quelle mesure elle est à même d'étayer l'origine anthropique des forêts à Sarcolaenanceae et Dypsis spp (Arecaceae) comme formations secondaires âgées résultant d'une longue reconstitution naturelle du milieu après défrichement ou passage du feu dans la région. Dans ce contexte, il nous semble primordial d'analyser le processus du tavy, d'évaluer son rôle dans la société paysanne, d'essayer de comprendre les raisons de sa persistance, afin de pouvoir envisager des alternatives et des solutions pour conserver les reliques des forêts du Sambirano, sans pour autant prétériter le développement du milieu humain.

\section{HISTORIQUE DE LA PRESQU'ÎLE D'AMPASINDAVA}

IMPORTANCE DANS LA CIRCULATION MARITIME DEPUIS LE

$X$ SIĖCLE. Les régions nord et nord-ouest de Madagascar sont riches en éléments historiques concernant la première implantation humaine dans l'île. D'après les découvertes effectuées dans les grottes de Lakaton'i Anja (Montagne des Français) et d'Ambohiposa (Vohémar) (Dewar et al. 2013), les humains étaient présents depuis longtemps (du Ile au Vle siècle) à Mada- 
gascar. Selon Wright et al. (2005), le port commercial le plus ancien sur les côtes de Madagascar se trouve à Mahilaka, dans la baie d'Ampasindava ; il atteint son apogée entre le Xle et le XIVe siècle grâce au commerce des Arabes avec la côte Est-Africaine. L'arrivée des premiers Européens date du 10 août 1500 avec le Portugais Diego Diaz, un des capitaines de la flotte de Pedralvarez Cabral en route pour les Indes, séparé de ses compagnons à cause d'une tempête. II baptise cette terre pour lui inconnue 'Saint Laurent', le nom du saint pour cette date (Grandidier 1902). A la suite de cette découverte, une première reconnaissance a été effectuée par les Portugais entre 1506 et 1507, et des établissements commerciaux se sont installés dans le Nord-ouest de Madagascar.

À cette époque, la région de la presqu'île d'Ampasindava était donc souvent visitée par les navigateurs grâce en particulier à la nature des côtes, adéquate pour le mouillage des grands bateaux et des boutres. La situation de la presqu'île d'Ampsindava en a fait un des grands carrefours pour les Austronésiens, les Arabes et les Anglais, escale idéale entre l'Afrique et I'Asie (Grandider 1902, Beaujard 2007). On rencontrait déjà alors à Anorotsangana des immigrés africains (à cause du trafic lié à l'esclavage), originaires de Malindi (Kenya), de Mogadicho (Somalie) et du Mozambique (Grandidier 1902). Les Russes sont également passés dans la région en 1905, faisant des escales dans le nord, d'où le nom "Baie des Russes ». La presqu'île d'Ampasindava a donc fait l'objet d'un intense trafic maritime depuis plus de cinq siècles.

PREMIER SIÈGE ROYAL DES SAKALAVA DANS LE NORD-OUEST DE MADAGASCAR. La région septentrionale de l'île a connu sa première implantation humaine au IVe siècle, et sur la côte nord-ouest dans la baie d'Ampasindava entre le Xe et le Xlle siècle (Wright et al. 2005). Cette présence humaine ancienne a été confirmée par des fouilles archéologiques dans la baie à l'ouest de l'archipel d'Ambariotelo (Ampasindava), à Nosy Be et à Mahilaka, ainsi que des récits historiques (Poirier 1948, Radimilahy 1998, Wright et al. 2005). Si cette zone était animée par les voyages des marins et des commerçants, elle l'était aussi par la migration des populations indigènes, causée par des conflits internes entre les Merina (ethnie des hautes terres centrales) et les Sakalava (ethnie de la région ouest), et lorsque Madagascar était sous la domination coloniale de la France (Ballarin 2007).

Après le déclin des Portugais vers 1530, Madagascar a entamé des relations avec les Anglais par l'intermédiaire du royaume Merina. Cette situation a marqué un tournant pour I'histoire du peuple malgache et pour le pays, car elle a favorisé et affermi I'ambition du pouvoir royal Merina et sa politique expansionniste. À partir de 1817, le royaume Merina, sous le souverain Radama I, a cherché à conquérir l'ensemble de la Grande Île. Pour y parvenir, et afin que le royaume Merina soit reconnu comme le royaume de Madagascar, il a accepté les conditions imposées par les Anglais, notamment l'abolition de la traite extérieure des esclaves. Par la suite, le roi Radama I a cherché à accaparer toutes les zones importantes de la Grande île en utilisant des moyens politiques et militaires. En 1824, le royaume Sakalava de la région ouest de Madagascar (région de Mahajanga) était conquis, avec pour conséquence la fuite des dynasties locales Maroseranana (Deschamps 1959), qui étaient formées par les troupes de Zafimbolamena et de Zafimbolafotsy. À cause de la forte pression des Merina, les Zafimbolamena se sont enfuis vers le nord et se sont installés sur la presqu'île d'Ampasimena, sur le territoire d'Anorotsangana, presqu'île d'Ampasindava. La première résidence royale des Sakalava dans le Nord de Madagascar a donc été établie sur la presqu'île d'Ampasindava, avec une concentration de la population dans la zone d'Anorotsangana (Amicale des Journalistes et Écrivains Français de Madagascar 1942).

Plus tard, en 1832, une scission divisa les Bemihisatra en deux royaumes distincts : I'Ampanjakabe (roi) Andriantsoly partit à Mayotte et fonda un nouveau royaume, tandis que la reine Oantitsy fonda un royaume sur le littoral de la presqu'île d'Ampasindava. Sa jeune sœur Tsiomeko lui succéda en 1836. Durant cette période, les hostilités entre Sakalava et Merina prirent un nouveau tournant qui marqua I'histoire de la presqu'île d'Ampasindava. L'armée du royaume Merina, forte de deux mille hommes qui furent envoyés par le souverain, était devenue trop puissante pour les Sakalava, et les envahisseurs cherchèrent à capturer Tsiomeko, la reine des Sakalava Bemazava et ses conseillers Antalaotra et Tsimandroho (Ballarin 2000). Ces derniers choisirent de se réfugier au centre de la presqu'île, notamment dans les grands massifs forestiers. C'est pour cette raison que les indigènes considèrent aujourd'hui encore ces massifs comme sacrés, et en particulier le massif de Bongomirahavavy (qui signifie 'montagnes sœurs') qui a probablement servi de cachette aux sœurs Oantitsy et Tsiomeko.

Lorsqu'en 1839 les hostilités reprirent, une grande partie des Bemihisatra, menés par leur reine Tsiomeko, se réfugia sur l'île de Nosy Be. Comme la France, rivale de l'Angleterre dans la conquête de Madagascar, était attirée par la richesse et la beauté de l'île de Nosy Be, la reine des Bemihisatra passa un accord avec la France en lui cédant son territoire, en échange d'une protection de son peuple contre les Merina (Ballarin 2000). Sur la presqu'île d'Ampasindava, cette guerre avec le royaume Merina est restée gravée aussi bien dans les paysages que dans la mémoire et les traditions des gens avec le respect des valeurs culturelles et coutumières des zones à importance historique (Bongomirahavavy, Andranomatavy, Marotony, Ambariotelo ou d'Ambarijeby). Ces milieux sont sacrés pour les natifs de la région de par les liens qui les unissent au royaume Sakalava, en tant que massifs forestiers qui servirent de refuge pendant les guerres. Cette notion du sacré a été renforcée par des règles coutumières qui se sont transformées en interdits ou fady. Parmi ces interdits, les plus importants portent sur l'interdiction pour les Merina de fréquenter la région ainsi que sur la conservation de certaines forêts dont : (i) les Merina n'ont pas le droit de circuler dans les massifs sacrés ; (ii) le dialecte merina ne doit pas être parlé à l'intérieur des forêts des massifs sacrés; (iii) un habit de couleur rouge ou noir (couleur des armées merina) ne doit pas être porté dans les forêts sacrées; et (iv) il est interdit de couper, défricher ou de pratiquer le tavy dans les forêts sacrées.

SYSTĖME ÉCONOMIQUE COLONIAL, FLUX MIGRATOIRE ET EXPLOITATION DES FORÊTS. Le nord-ouest de Madagascar, avec son climat favorable, ses grandes plaines alluviales et ses sols souvent favorisés par leur nature volcanique, a compté parmi les points économiques stratégiques pendant la période coloniale. Plusieurs régions de grande importance économique sont passées sous protectorat français suivant l'annexion du 6 août 1896 dont Nosy Be, la presqu'île d'Ampasindava, la plaine du Sambirano, les plaines de l'Ifasy, de la Mahavavy et du Mananjeba. Une exploitation massive des forêts a d'abord eu lieu pour 
I'extraction des ressources en bois et des plantes à caoutchouc, ainsi que pour l'aménagement de terrains pour les plantations de rente. Pour développer le système de production économique coIonial, les Français ont imposé un système de flux migratoire pour augmenter la main d'œuvre dans le domaine du Sambirano. Madagascar était alors subdivisé en deux types de zones, les zones de départ qui étaient sources de mains d'œuvre et les zones d'arrivée qui étaient les zones de production. Le district d'Ambanja était une zone d'arrivée, où se sont implantées de grandes sociétés agricoles. De nombreuses personnes venant de différentes régions arrivèrent dans la zone, tels les Antandroy en provenance de la région Androy, les Betsileo de la région de Fianarantsoa, les Tsimihety des régions de Mandritsara et d'Antsohihy, et les Antemoro de la région sud-est de Madagascar (Waast 1972). En conséquence, la population active de la région a très vite connu une forte croissance.

Suite aux travaux forcés imposés par les colons, des révoltes eurent lieu et causèrent la fuite de nombreuses personnes qui s'installèrent dans les forêts et commencèrent à exploiter le milieu (Waast 1972). C'est à partir de cette période que le massif du Manongarivo, les forêts du Sambirano ainsi que les forêts de la zone est de la presqu'île d'Ampasindava ont connu de plus en plus le tavy, malgré l'existence de lois du 28 août 1913 interdisant les feux en forêt et les feux de brousse.

DE L'INDÉPENDANCE À LA PÉRIODE ACTUELLE. Depuis I'indé pendance, la presqu'île est devenue un grand fournisseur en riz, en bois de construction et en charbon pour Nosy Be et Ambanja. Ceci est dû à sa grande richesse en forêts et en mangroves non protégées et accessibles à tous, à l'inverse des régions de Nosy Be et d'Ambanja où les forêts restantes sont limitées aux aires protégées (Lokobe et Manongarivo). Cette grande disponibilité des ressources naturelles attire les paysans de différentes régions, notamment les Tsimihety de la région Sofia (au sud-est de la presqu'île). En plus, pendant la saison sèche, la presqu'île d'Ampasindava accueille un grand nombre d'immigrants, venus principalement pour chercher un emploi comme défricheurs. Ces défricheurs (appelés localement Karama tetika) sont en général des Tsimihety payés 5000 Ariary/jour. Leur présence a pour conséquence d'augmenter non seulement le taux de surface défriché par année, mais aussi la densité de la population. Arrivés à I'origine comme employés saisonniers, beaucoup d'entre eux resteront après la fin de la saison des défrichements et commenceront à faire leur propre tavy. Ce cas est souvent observé dans les communes d'Ambaliha et de Bemanevika.

Par la présence de la route nationale 6, la population des communes d'Ankaramy, d'Anjiabory, et d'Ankingameloka ont connu une hausse considérable. Puisque la forêt de Manongarivo a un statut de Réserve spéciale, et est protégée contre toute forme d'activité anthropique, les paysans à la recherche de nouvelles terres à défricher se sont déplacés de plus en plus vers le nord-ouest et sont arrivés dans la région d'Antsirabe et d'Ambaliha. En conséquence de ces déplacements de population, une nouvelle délimitation administrative a élevé le village d'Ambaliha au rang de commune rurale (CR), celle-ci s'ajoutant aux CR d'Anorotsangana, d'Antsirabe et de Bemanevika., La pratique du tavy s'est donc intensifiée et a détruit les forêts des massifs d'Ankobabe, de Bezavona, d'Ambilanivy et une partie de celui de Bongomirahavavy.

La crise politique de 2009 a également contribué à la trans- formation du paysage de la presqu'île d'Ampasindava. Les industries et les grandes entreprises de la région nord-ouest de Madagascar telles que SIRAMA (industrie de production de sucre de Madagascar), MAGRO (Malagasy Grossiste) de Nosy Be, d'Ambanja et d'Ambilobe ont choisi de fermer leurs portes ou ont été obligées de suspendre leurs activités. De nombreux employés se sont retrouvés au chômage et les familles ainsi en difficulté se sont tournées vers la presqu'île d'Ampasindava pour pratiquer le tavy, l'exploitation des bois, ou la pêche, simplement pour survivre, accélérant l'intensité de la dégradation de l'environnement de la région au cours de ces cinq dernières années.

\section{LE TAVY : ACTIVITÉ PRINCIPALE DE LA POPULATION LOCALE}

IMPORTANCE DU RIZ ET DU TAVY DANS LA VIE QUOTIDIENNE

DES PAYSANS. Comme dans tous les milieux ruraux mal-

gaches, le zébu et le riz représentent les deux grands signes de richesse des paysans. Sur la presqu'île d'Ampasindava, l'élevage extensif est peu pratiqué et le nombre moyen de têtes de bétail par ménage est limité : entre deux et cinq zébus. Le riz en revanche occupe une grande place tant dans l'économie que dans la constitution des rangs des classes sociales des paysans. La quantité de riz produite fait la richesse d'une personne ou d'une famille donnée. Un paysan qui a eu la chance de faire un tamborôho ou valintanana (une activité sollicitée par un agriculteur qui a pu produire une grande récolte, et demande de ce fait aux voisins ou aux villageois de lui donner une aide pour la moisson sans rétribution mais seulement en échange de la nourriture pendant les jours de travail) et qui a offert un zébu aux membres de la société pendant les jours de la moisson jouit d'une haute considération. Ainsi, celui qui a eu le plus grand tôha ou riha (une case construite spécialement pour stocker le paddy et dont la taille dépend de la quantité de la production annuelle) devient une personne modèle et respectée dans la société. Cette importance sociale de la production du riz aggrave le phénomène de la déforestation sur la presqu'île d'Ampasindava, car elle génère une concurrence entre les paysans et augmente considérablement le rythme du tavy. Par ailleurs, le tavy constitue un moyen pour s'approprier des terrains. À cause de la non-application ou le non-respect des lois de législation forestière la forêt primaire est considérée par les paysans comme une ressource communautaire. Par un choix avisé des zones à défricher, l'agriculteur assure la qualité de la production et augmente son pouvoir foncier et celui de sa descendance. Cet aspect foncier de la pratique du tavy concourt également à favoriser la déforestation.

Le tavy est pratiqué par tous les paysans, surtout par les hommes. II est un moyen de montrer une capacité d'autonomie au sein de la société. En effet, celui qui est capable de réaliser et d'entretenir à lui seul son tavy devient un homme respectable. C'est pourquoi, dès l'âge de 14-15 ans, les jeunes hommes commencent à avoir leur propre tavy en plus de celui partagé avec la famille. Selon le nombre de personnes actives par ménage et le pouvoir de chacun à défricher la forêt, la superficie d'un tavy peut varier de $900 \mathrm{~m} 2$ à 10000 m2 par ménage. Une parcelle étant généralement cultivée pendant deux années successives avant la mise en jachère, cette surface correspond à la surface défrichée tous les deux ans. Les parcelles défrichées sont des milieux riches en biomasse, comme des friches et très souvent des forêts secondaires, voire primaires dans le but d'avoir beaucoup de combustion lors de l'incinération et d'enrichir ainsi le sol en 
substances nutritives. Si l'espace de production vient à manquer les paysans se tournent vers des forêts secondaires de plus en plus jeunes. Par ailleurs, si les forces manquent pour défricher des forêts âgées, les paysans choisissent les parcelles en jachère, plus faciles à travailler.

CHOIX DES PARCELLES ET PRATIQUE DU TAVY. Dans le sys-

tème d'agriculture itinérante, la fertilité du sol est un élément primordial dans le choix des parcelles de culture. Les paysans identifient les milieux fertiles et propices au tavy selon quelques critères, notamment les espèces indicatrices du type de sol et de la structure du substrat, la présence et l'abondance de turricules (dû à l'activité des vers de terre), ainsi que la taille des arbres. Sur la presqu'île d'Ampasindava, la présence de lianes comme Merremia peltata (L.) Merr (Convolvulaceae) ou Vahimbalegny. (Ampelocissus elephantina Planch. - Vitaceae) ou Menakibongana, et I'abondance d'arbres comme Parkia madagascariensis R.Vig. (Fabaceae) ou Fanamponga, Khaya madagascariensis Jum. \& H. Perrier (Meliaceae) ou Sandramiramy, Calophyllum sp. (Calophyllaceae) ou Lintanona, ou Lazalaza ou Mongy, tous deux Croton spp. (Euphorbiaceae), qui indiquent une meilleure qualité du sol, font présager d'une grande production. Ces milieux sont préférés pour le tavy, et d'autant plus s'ils ne sont pas loin du village et proches de cours d'eau. Les forêts les moins prisées sont celles qui se développent sur des sols minces ou celles où l'abondance en Rinorea arborea (Violaceae) ou Hazondomohina, Homalium micranthum (Boivin ex Tul.) O. Hoffm. (Salicaceae) ou Janganito et Scleria boivinii Steud. (Cyperaceae) ou Filelatra est élevée car elles reflètent des conditions jugées défavorables à la culture de riz. Les forêts riches en Martellidendron sp. (Pandanaceae) sont également évitées à cause de la présence de nombreuses racines échasses et épines, très difficiles à défricher.

La production du tavy est importante lors de la première année de culture, mais présente une baisse considérable l'année suivante. En effet, il y a une perte en éléments nutritifs du sol par le phénomène de lessivage et de dénitrification, ainsi qu'un accroissement de la concurrence des mauvaises herbes. Lorsque les jachères et les friches ont été au repos pendant 15 à 30 ans, les paysans les reconsidèrent lorsque les espèces indicatrices redeviennent abondantes, indiquant que le sol a retrouvé ses qualités pour la production de riz.

PRODUCTION DE RIZ DANS L'ÉCONOMIE DE SUBSISTANCE. La production de riz par ménage dépend de plusieurs facteurs : le type de parcelle (type de formation, historique), la qualité du sol, et la quantité et la répartition des précipitations. Dans la commune rurale de Bemanevika, la production moyenne annuelle communale est comprise entre 1600 et 1900 tonnes de riz blanc (Missouri Botanical Garden 2014). La consommation moyenne journalière d'une personne active est quant à elle estimée à 0,72 $\mathrm{kg}$, ce qui représente une consommation moyenne annuelle de 1,303 tonne par ménage (avec 5 personnes/ménage). D'après ces chiffres, la quantité de la production serait donc suffisante pour I'autoconsommation. Cependant les paysans sont obligés de vendre une partie de leur récolte pour acquérir divers produits manufacturés, réduisant ainsi les réserves dont ils ont besoin pour boucler l'année. Ils doivent ainsi surmonter une période de soudure annuelle de janvier à mars, durant laquelle ils sont obligés d'acheter du riz, et se tournent vers l'exploitation des bois afin de générer les revenus nécessaires.
Le calendrier agricole des paysans est chargé (Tableau 1), seIon les données des enquêtes collectées. La culture du riz occupe la saison des pluies, et le défrichement et la mise à feu sont réalisés pendant la saison sèche. Entre ces deux saisons, pendant la période de soudure, l'exploitation forestière atteint son maximum. Pour les paysans vivants sur les côtes, la production de charbon, destinée au marché de Nosy Be, est intensive pendant les mois d'août et de septembre, et baisse pendant la saison des pluies.

LE TAVY, UN PROBLÈME NON RÉSOLU À MADAGASCAR.

Plusieurs dirigeants malgaches, anciens et récents (rois, présidents ou ministres), ont tenté de résoudre les problèmes liés au tavy. De nombreux organismes non gouvernementaux ont également déployé beaucoup d'efforts pour gérer ce problème et proposer des solutions à l'Etat (e.g., EPB-BEMA, SAGE, TAMS). Mais la forêt continue à diminuer d'année en année, et la pauvreté augmente toujours.

Le roi Andrianamponimerina (1787-1810) considérait les forêts comme un patrimoine, source de biens pour les orphelins, les veuves et les malheureux, qu'il fallait protéger, car ces derniers n'avaient rien à vendre ni à manger (Lavauden 1939). Les lois de réglementation du code 305 de la reine Ranavalona (en 1881) (Julien 1932) se sont efforcées de donner une base légale à cette intention de protection en interdisant le défrichement. Pendant la colonisation, le décret de 1913 a renforcé cette interdiction, en proscrivant les feux et particulièrement les tavy, ou en les soumettant à un régime d'autorisation administrative préalable. Après une première période où ce système a été mis en place et respecté, un certain laxisme s'est développé, et ce surtout depuis que les colons, impliqués dans la première guerre mondiale, n'avaient plus sur place les effectifs nécessaires.

Depuis la première république (1959-1975), le gouvernement a reconnu l'existence des défrichements abusifs. L'article 101 de Ranavalona a été mis à jour pour prendre en compte l'évolution démographique et l'état des forêts. Dans l'article 2 de l'ordonnance 62-121 qui porte à la modification de l'ordonnance 60-127, I'Etat malgache a défini le défrichement comme la suite des opérations destinées à permettre la mise en culture d'un terrain préalablement recouvert d'une végétation avec I'intention de l'incinérer après dessiccation pour y faire des plantations ou des semis d'ordre agricole. Le défrichement ainsi défini est considéré comme un délit et des réglementations furent mises en place pour le contrôler. Comme précisé dans l'article 3, il est interdit de procéder à des défrichements dans les forêts classées, les réserves naturelles, les parcs nationaux et les réserves spéciales, les périmètres de reboisement et de restauration, les stations forestières et les terres affectées au service des eaux et forêts. II est aussi interdit au niveau des versants des collines présentant une

Tableau 1. Calendrier des cultures et autres activités ajusté aux données climatiques (Station de Nosy Be)

\begin{tabular}{|c|c|c|c|c|c|c|c|c|c|c|c|c|}
\hline & \multicolumn{12}{|c|}{ Mois } \\
\hline & $\mathrm{VII}$ & VIII & $\alpha$ & $x$ & $\mathrm{Xl}$ & $\mathrm{X \| l}$ & 1 & $\|$ & III & N & $\mathrm{v}$ & $n$ \\
\hline $\begin{array}{l}\text { Précipitations } \\
(\mathrm{mm})\end{array}$ & 17,0 & 21,5 & 20,0 & 25,2 & 95,6 & 270,5 & 275,8 & 274,8 & 228,9 & 80,5 & 26,7 & 19,0 \\
\hline $\begin{array}{l}\text { Températures } \\
\text { moyennes }\left({ }^{\circ} \mathrm{C}\right)\end{array}$ & 25,2 & 25,5 & 25,9 & 27,6 & 27,8 & 27,8 & 27,7 & 27,7 & 27,8 & 27,7 & 27.0 & 26,8 \\
\hline Défrichement & ++ & ++ & +++ & +++ & & & & & & & & + \\
\hline Semis & & & & & 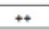 & $+4+$ & $+4+$ & & & & & \\
\hline $\begin{array}{l}\text { Désherbage } \\
\text { manuel }\end{array}$ & & & & & & & + & $+*+$ & + & & & \\
\hline Moisson & & & & & & & & & & + & $+\infty$ & ++ \\
\hline $\begin{array}{l}\text { Exploitation de } \\
\text { bois }\end{array}$ & ++ & ${ }^{++}$ & + & + & + & + & +++ & +++ & +++ & & & ++ \\
\hline $\begin{array}{l}\text { Charbonnage } \\
\text { (Mangroves) }\end{array}$ & ++ & +++ & +++ & + & + & + & + & + & $*+*$ & $+*+$ & + & + \\
\hline
\end{tabular}


pente supérieure à $50 \%$, dans les dunes littorales, sur les berges des rivières et cours d'eau sur une largeur de $20 \mathrm{~m}$ à partir de la limite des plus hautes eaux, dans leurs coudes et méandres, et aux abords des ouvrages d'art. Ces lois furent confirmées par le Président Didier Ratsitaka suivant la loi n 97-017 du 8 août 1997 portant sur la révision de la législation forestière (J.O. $n^{\circ} 2449 \mathrm{du}$ 25.08.94, p. 1717), chapitre VI, Art.24 en mentionnant que les dispositions de l'ordonnance $n^{\circ}$ 60-127 du 3 octobre 1960 fixant le régime des défrichements et des feux de végétation restaient applicables.

En théorie, ces différentes lois et ces décrets seraient amplement suffisants pour protéger les forêts et les aires protégées de Madagascar, s'ils étaient appliqués correctement. Les analyses spatiales et temporelles des changements de la couverture forestière ont montré que le tavy commence toujours depuis les environs des zones d'habitation, qui sont principalement aux bords des cours d'eau. Ensuite, le tavy se pratique à des altitudes de plus en plus élevées, et sur des terrains de plus en plus accidentés. Les analyses faites par Gautier et al. (1999) sur le pourtour de la réserve spéciale de Manongarivo, dans le domaine du Sambirano, ont démontré qu'avant 1987 la majorité des tavy étaient établies sur des pentes comprises entre 0 et $50 \%$, mais que depuis 1995, ont les trouvait de plus en plus fréquemment sur des pentes plus fortes, atteignant $70 \%$ à $90 \%$.

\section{CONSÉQUENCES DES FLUX MIGRATOIRES SUR LA TRANSFORMATION DU PAYSAGE}

Les données historiques présentées ici, mises en regard avec les observations menées sur le terrain sur la structure et la composition des forêts, permettent d'avancer que la dégradation des forêts de la presqu'île d'Ampasindava a connu une ampleur importante déjà à une époque ancienne. Avec le développement du circuit maritime reliant I'Indonésie, I'Europe, I'Arabie et I'Afrique, la zone nord-ouest de Madagascar, notamment les régions d'Ampasindava, de Mahilaka et de Nosy Be sont devenues un important carrefour pour les commerçants et les marins et le milieu naturel de la région a connu une présence humaine croissante. Des fouilles archéologiques effectuées dans la région de Mahiaka et d'Ampasindava ont permis de trouver des grains de riz carbonisés parmi d'autres restes de constructions et divers matériaux (Wright et al. 2005). En l'absence d'une topographie favorable à la culture irriguée, ceci nous permet de penser que la pratique de la culture du riz par l'agriculture itinérante sur brûlis se pratiquait dans la région à l'ère de l'expansion du commerce au Xe siècle. L'agriculture itinérante du riz sur brûlis ou tavy a ainsi commencé à marquer le paysage.

Au XVIe siècle, dans la région d'Anoratsangana, les troupes portugaises ont chassé les esclaves africains échappés par différents moyens, notamment le feu pour brûler villages plantations. Les escales des commerçants et la fuite des esclaves ont été à I'origine du développement des premières activités humaines (agriculture et chasse) dans la région. Le défrichement a commencé à transformer le paysage de la presqu'île et s'est intensifié avec l'augmentation de la population. Cette transformation du paysage a commencé au sud-ouest de la presqu'île, dans la zone d'Anorotsangana, et s'est déplacée progressivement vers le nord.

Au début du XIXe siècle, la guerre entre les Merina et les Bemihisatra a apporté sa contribution à la transformation du paysage. Fuyant les troupes Merina, les Bemihisatra sont arrivés sur la presqu'île d'Ampasindava depuis Ampasimena et ont continué à avancer vers Ambarijeby, Ambodivoanio, Andrahibobe, situées sur le territoire d'Anorotsangana et sur le littoral nord (Baré 1980) où ils ont exploité le milieu. Le tavy a donc pris de l'ampleur avec I'augmentation de la population. C'est encore le cours de cette histoire qui a sacralisé ces grands massifs de Bongomirahavavy et d'Andranomatavy. Le concept de la conservation des massifs forestiers s'est développé, en l'honneur des services et de la protection qu'ils avaient fournis aux Sakalava. Ce statut a longtemps assuré la protection de ces milieux contre toutes formes de menaces et pressions anthropiques, permettant la conservation de la structure et de la physionomie originelles des forêts denses humides, qu'on peut ainsi qualifier de végétation climacique. Des valeurs culturelles se sont ainsi transmises de génération en génération, avec la notion du respect du fady. C'est la raison pour laquelle les forêts de ces massifs sont restées intactes et forment actuellement l'essentiel des reliques des forêts primaires de la presqu'île d'Ampasindava.

Au fil de cette succession d'événements, la région la plus touchée par la déforestation a été la partie ouest de la presqu'île. La déforestation a commencé dans la partie sud-ouest de la presqu'île. La région d'Anorotsangana en a été le point de départ, et la pratique du tavy s'est étendue de plus en plus au nord-ouest vers Betsiriry et Komamery jusqu'à Marotony et Maroariva (Figure 1). Le défrichement et l'incendie ont détruit d'importantes surfaces de forêts primaires, laissant ensuite place au processus de la succession végétale. De par l'arrivée progressive des paysans, le paysage est devenu de plus en plus hétérogène à cause de la dégradation et et de la fragmentation des forêts, dont les zones les plus touchées sont surtout situées dans la partie ouest de la presqu'île.

Pendant la colonisation, une population relativement clairsemée et l'application des lois contre l'exploitation des forêts par les colons sont probablement à l'origine d'une baisse du rythme de la transformation du paysage. II est ainsi vraisemblable que les milieux dénudés ou exploités pendant ces périodes se sont ensuite reconstitués petit à petit pour donner les formations forestières secondaires actuellement observées.

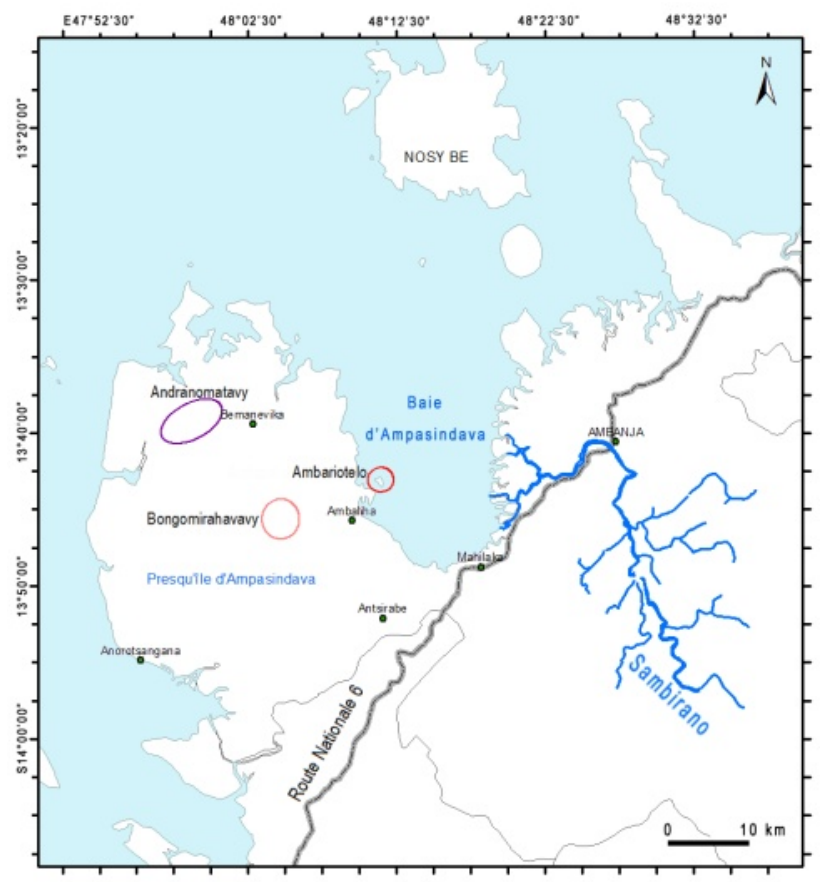

Figure 1. La presqu'île d'Ampasindava dans le domaine du Sambirano. 
Pour ces raisons, l'hypothèse émise que nous avançons ici d'une ancienne exploitation et destruction massive du milieu semble être la seule qui puisse donner une explication acceptable de la structure et de la composition floristique de la forêt de la partie occidentale de la presqu'île d'Ampasindava. Les forêts à Sarcolaenaceae et Dypsis spp doivent être considérées comme des formations secondaires âgées en plein développement. Leur composition et leur structure, bien que différentes de celles des forêts primaires, en font des sites potentiels de tavy pour les générations actuelles.

D'après les données des analyses diachroniques des images satellitaires (1991-2013) la presqu'île d'Ampasindava présente actuellement un rythme élevé en perte de forêt (secondaires âgées et primaires) de 1500 ha par année (Tahinarivony 2014). Si la population malgache présente une augmentation moyenne annuelle de 3\% (INSTAT 2014), celle de la région de la presqu'île d'Ampasindava est estimée au-dessus de la moyenne en raison d'un fort taux de natalité et de l'arrivée d'immigrants à la recherche des terres cultivables (Missouri Botanical Garden 2014). La croissance démographique endogène, mais surtout l'immigration, ont eu pour conséquence la réduction importante des forêts rendant le choix des parcelles à défricher pour le tavy de plus en plus difficile. Pour ces raisons, le temps de jachère diminue, et la forêt et le sol n'ont plus le temps de se reconstituer pleinement entre deux cycles culturaux. Les paysans sont donc contraints de défricher des parcelles de plus en plus éloignées des villages, atteignent des altitudes de plus en plus élevées et mises en place sur des pentes de plus en plus fortes (Gautier et al. 1999). Ils vont par ailleurs fabriquer des cases près de leur tavy pour se rapprocher de leurs zones de production.

La forte dépendance des paysans vis-à-vis des ressources forestières conjuguées à cette réduction de leur surface font donc peser une menace de plus en plus importante sur les massifs forestiers résiduels. Les habitants de la presqu'île sont écartelés entre le respect du sacré et la volonté de produire le riz nécessaire à leur subsistance et à financer leurs besoins. Beaucoup ont commencé ainsi à transgresser les interdits en défrichant des massifs traditionnellement protégés, en particulier les immigrants qui se sentent moins concernés par ces interdits coutumiers d'une ethnie à laquelle ils n'appartiennent pas.

\section{CONCLUSION}

LA PRESQU'ÎLE D'AMPASINDAVA DIX ANS APRĖS LA VISION

DURBAN. Par la vision Durban en 2003 qui vise à tripler la superficie des aires protégées, allant de 1,7 millions d'hectares à 6 millions d'hectares, une nouvelle directive a été émise, portant sur la mise en place d'un système de Nouvelles Aires Protégées de Madagascar (NAP). Différents spécialistes de plusieurs disciplines ce sont rassemblés pour analyser les données de plusieurs groupes d'espèces, comme les mammifères, les reptiles, les amphibiens, les poissons, certains invertébrés et la flore (Kremen et al. 2008, Allnutt et al. 2009). Les résultats issus de ces analyses ont été combinés avec les données des zones clés de la conservation de la biodiversité et celles des changements climatiques, les analyses ont été affinées et sont devenues de plus en plus pertinentes (Eken et al. 2009) suivant l'approche de zonation de Moilanen et al. (2009). Leur étude a abouti ensuite à la publication des cartes des zones potentielles de conservation qui a montré l'importance écologique de la presqu'île d'Ampasindava, en plus de son degré de priorisation pour la conservation. Mal- heureusement, entre 2009 et 2013, le pays a traversé une longue crise, ralentissant ou interrompant certains projets de conservation dans le pays.

Il est à présent temps de relancer cette dynamique en développant un projet de conservation innovant, qui prenne en compte d'une part la problématique socio-économique propre à la région, et d'autre part la préexistence d'interdits coutumiers. Avec une démographie en augmentation, il est douteux que la pratique du tavy soit à même de répondre seule aux besoins croissants des populations. II faut donc envisager des solutions qui tendent à sédentariser la production de riz dans des parcelles permanentes. Pour une sauvegarde durable des forêts existantes, une politique de conservation durable doit impérativement se baser sur les interdits coutumiers existants, en renforçant le rôle des acteurs autochtones dans leur application (conservation participative). II serait par ailleurs salutaire d'envisager un programme d'éducation environnementale qui mette en valeur en parallèle le rôle écologique de la forêt mais aussi sa valeur culturelle pour la communauté locale.

\section{REMERCIEMENTS}

Cette recherche a été effectuée dans le cadre de l'étude de la biodiversité et de la transformation du milieu de la presqu'île d'Ampasindava, financé par la Fondation Vontobel à Zürich (Suisse). Notre reconnaissance s'adresse à tous les rapporteurs et éditeurs du présent article. Nous remercions également les Conservatoires et Jardin Botaniques de la Ville de Genève et I'Université d'Antananarivo.

\section{RÉFÉRENCES}

Allnutt, T. F., Asner, G. P., Golden, C. D. \& Powell, G. V. 2013. Mapping recent deforestation and forest disturbance in northeastern Madagascar. Tropical conservation Science 6: 1-15.

Amicale des Journalistes et Écrivains Français de Madagascar. 1942. Cahiers Malgaches 1941-Centenaire du Rattachement à la France des îles : Nossi-Bé, et Mayotte. Imprimerie Officielle, Antananarivo.

Ammann, M. 2011. La Presqu'île d'Ampasindava (Nord-Ouest de Madagascar) Une Région mal Connue et Menacée, Capitale pour la Compréhension de la Mise en Place de la Flore du Domaine du Sambirano. Unpubl. Mémoire de Master, Université de Genève, Suisse

Aubert, S., Razafiarison, S., Bertrand, A. 2003. Déforestation et Systèmes Agraires à Madagascar. Les Dynamiques des Tavy sur la Côte Orientale. $\mathrm{Cl}$ RAD/CITE/FOFIFA, Montpellier, Antananarivo.

Ballarin, M. P. 2000. Les Reliques Royales à Madagascar: Source de Légitimation et Enjeu de Pouvoir, XVIIIe-XXe siècles. Karthala, Paris.

Baré, J.-F. 1980. Sable Rouge : Une Monarchie du Nord-ouest Malgache dans I'Histoire. Harmattan, Paris.

Beaujard, P. 2007. L'Afrique de l'Est, les Comores et Madagascar dans le systèmemonde avant le XVle siècle. In: Madagascar et l'Afrique : Entre Identité Insulaire et Appartenances Historiques. D. Navitel et F. V. Rajaonah (eds.), pp 29-102. Karthala, Paris.

Cornet, A. 1974. Essai de Cartographie Bioclimatique à Madagascar. Notice Explicative $N^{\circ} 55$. ORSTOM, Paris. Disponible sur <http://horizon.documentation.ird.fr/exl-doc/pleins_textes/pleins_textes_5/notexp/06946.pdf>

Deschamps, H. 1959. Les Migrations Intérieures Passées et Présentes à Madagascar. Berger-Levrault, Paris. Disponible sur <http://horizon.documentation.ird.fr/exl-doc/pleins_textes/divers11-03/15847.pdf>

Dewar, R. E., Radimilahy, C., Wright, H. T., Jacobs, Z., Kelly, G. O. \& Berna, F. 2013. Stone tools and foraging in northern Madagascar challenge Holocene extinction models. Proceedings of the National Academy of Sciences of the United States of America 110: 12583-12588. (doi:10.1073/pnas.1306100110) 
Du Puy, D. \& Moat, J. 1998. Vegetation mapping and biodiversity conservation in Madagascar Geographical Information Systems. In: African Plants: Biodiversity Taxonomy and Uses. J. Timberlake \& S. Kativu, (eds.), pp 245-251. Royal Botanic Gardens, Kew.

Eken, G., Bennun, L., Brooks, T. M., Darwall, W., Fishpool, L. D. C. et al. 2004. Key biodiversity areas as site conservation targets. Bioscience 54,12 : 1110-1118. (doi:10.1641/0006-3568(2004)054[1110:KBAASC]2.0.CO;2)

de Flacourt, E. 1661. Histoire de la Grande Isle Madagascar. Avec une Relation de ce qui s'est passé en Années 1655, 1656 et 1657. Pierre l'Amy, Paris. Disponible sur <http://ow.ly/QMBP7>

Gautier, L., Chatelain, C. et Spichiger, R. 1999. Déforestation, altitude, pente et aires protégées : une analyse diachronique des défrichements sur le pourtour de la réserve spéciale de Manongarivo (NW Madagascar). In: African Moutain Development in a Changing World. H. Humi \& J. Ramamonjisoa (eds), pp 255-279. African Moutains Associations, Antananarivo.

Goodman, S. M. \& Benstead, J. P. 2005. Updated estimates of biotic diversity and endemism on Madagascar. Oryx 39, 1: 73-77. (doi:10.1017/S0030605305000128).

Grandidier, A. 1902. Histoire de la découverte de l'île de Madagascar par les Portugais. Revue de Madagascar $1: 34-54$.

Humbert, H. 1955. Les territoires phytogéographiques de Madagascar. Leur cartographie. Année Biologique 31(5-6): 439-448.

Humbert, H. et Cours Darne, G. 1965. Carte Internationale du Tapis Végétal et des Conditions Ecologiques. Baie d'Ampasindava. Institut Français de Pondichéry, Pondichéry.

Institut National de la Statistique. 2014. Madagascar en chiffre. Disponible : http://instat.mg/madagascar-en-chiffres/\#demographie

Julien, G. 1932. Code des 305 articles 1881. Instructions aux Sakaizambohitra. Règlements des Gouverneurs de I'Imerina. Imprimerie Officielle, Antananarivo.

Kremen, C., Cameron, A., Moilanen, A., Phillips, S. J., Thomas, C. D. et al. 2008. Aligning conservation priorities across taxa in Madagascar with high-resolution planning tools. Science 320: 222-226. (doi:10.1126/science.1155193)

Lavauden, L. 1934. Histoire de la législation et de l'administration forestière à Madagascar. Revue des Eaux et Forêts 72: 949-960

Missouri Botanical Garden. 2014. Inventaire de Référence des Sites du Projet MRPA - Lot N 1 : Ampasindava-Galoko - DP NO 01 - 13 /DP/UCPE/MRPA. Rapport Final. Disponible sur <http://ow.ly/O6Zi4>

Moat, J. \& Smith, P. 2007. Atlas of the Vegetation of Madagascar. Royal Botanic Gardens, Kew.

Moilanen, A., Wilson, K. A. \& Possingham, H. P. 2009. Spatial conservation prioritization: quantitative methods and computational tools. Oxford University Press, Oxford.

Oberlé, P. (ed.) 1981. Madagascar, un Sanctuaire de la Nature. Lechevalier SARL, Paris.

Poirier, C. 1948. Réflexion sur les ruines de Mahilaka situé au fond de la baie d'Ampasindava. Bulletin de l'Académie Malgache 28: 97-107.

Radimilahy, C. 1998. Mahilaka: An archaeological investigation of an early town in northwestern Madagascar. Studies in African Archaeology 15. Disponible sur <http://www.arkeologi.uu.se/digitalAssets/32/32403_3mahilika.pdf>

Razafy Fara, L. 2004. Les intérêts des différents acteurs dans la gestion des ressources naturelles forestières. Étude de cas dans la région de Beforona, est de Madagascar. Schweizerische Zeitschrift für Forstwesen 155, 3-4: 89-96. (doi:10.3188/szf.2004.0089)

Scales, I. R. 2014. The drivers of deforestation and the complexity of land use in Madagascar. In: Conservation and Environmental Management in Madagascar. I. R. Scales (ed.), pp 105-125. Routledge, London and New York.

Tahinarivony, A. J. 2010. Caractérisation Écologique de la Végétation dans la Presqu'île d'Ampasindava, Cas de la Forêt des Massifs de Bongomirahavavy et de Betsitsika (Domaine du Sambirano), Région DIANA. Unpubl. Mémoire de D.E.A, Université d'Antananarivo.

Tahinarivony, A. J. 2014. Analyse Typologique et Cartographique de la Transformation du Paysage de la Presqu'île d'Ampasindava, Domaine du Sambirano (NW-Madagascar). Unpubl. Mémoire de Certificat en Géomatique. Université de Genève. Disponible en Matériel Supplémentaire.
Veyret, P. 1948. Une synthèse de géographie tropicale. « Gourou, P. 1947. Les Pays Tropicaux. Principes d'une Géographie Humaine et Économique. Paris, Presses Universitaires, 1947 , in - $8^{\circ}$, VIII + 196 p., 16 figures ». Revue de Géographie Alpine 36, 1: 178-181.

Vicariot, F. 1970. Le problème du tavy en pays Betsimisaraka (Madagascar). Analyse préliminaire. Cahier de I'ORSTOM, Série Biologie 14 : 3-12.

Waast, R. 1972. Le Développement de I'Agriculture Commerciale, de la Rente et du Salariat dans le Sambirano (Nord-Ouest Malgache). ORSTOM, Paris.

Wright, H. T., Radimilahy, C. et Allibert, C. 2005. L'évolution des systèmes d'installation dans la baie d'Ampasindava et à Nosy Be. Taloha 14-15. Disponible sur $<$ http://www.taloha.info/document.php?id=137>

\section{SUPPLEMENTARY MATERIAL.}

Available online only.

Figure S1. Carte simplifiée de la végétation de la presqu'île d'Ampasindava. Classification supervisée sur une image satellite de 2013, projetée sur une teinte relief. Les deux principaux blocs de forêt dense humide sempervirente primaire restants apparaissent au nord-ouest (Andranomatava) et au centre-est (Bongomirahavavy) et correspondent à des massifs sacrés. Le reste de la presqu'île est actuellement dominée par des mosaïques de cultures, de jachères et de jeunes forêts secondaires, à l'exception d'importantes zones de forêts secondaires âgées visibles dans l'ouest.

S2. Tahinarivony, A. J. 2014. Analyse Typologique et Cartographique de la Transformation du Paysage de la Presqu'île d'Ampasindava, Domaine du Sambirano (NW-Madagascar). Mémoire de Certificat en Géomatique. Université de Genève. 